In Proceedings of IEEE/ACM Int Conf on Automated Software Engineering (ASE 2008)

(c) IEEE 2008. Personal use of this material is permitted. However, permission to reprint/republish this material for advertising or promotional purposes or for creating new collective works for resale or redistribution to servers or lists, or to reuse any copyrighted component of this work in other works, must be obtained from the IEEE.

\title{
MaramaEML: An Integrated Multi-View Business Process Modelling Environment with Tree-Overlays, Zoomable Interfaces and Code Generation
}

\author{
Lei $\mathrm{Li}^{1}$, John Hosking ${ }^{1}$ and John Grundy ${ }^{1,2}$ \\ ${ }^{1}$ Department of Computer Science and ${ }^{2}$ Department of Electrical and Computer Engineering \\ University of Auckland, New Zealand \\ \{l.li,john,john-g\}@cs.auckland.ac.nz
}

\begin{abstract}
MaramaEML is an integrated support tool for the Enterprise Modelling Language (EML) built using the Eclipse based Marama framework. It provides support for multiple visual notations including: the Business Process Modelling Notation (BPMN); the EML treebased, multi-layer hierarchical service representation; fisheye zooming capabilities; automatic BPEL code generation; and inter-notation mapping.
\end{abstract}

\section{Enterprise Modelling Language}

Most current approaches to modelling complex business processes fail to scale for large processes and organizations. Key issues include: (1) cobweb and labyrinth problems exhibited by conventional box and line metaphors and large numbers of hidden dependencies introduced by compartment-based modularity (e.g. BPMN, FormChart, BioOpera and ZenFlow [1][3][8][9]); (2) inefficiencies in presenting multi-level abstractions of business processes (e.g. WTD and T-Web systems [6]); (3) notations too complex for a business user to learn and use (e.g. UML [2]); and (4) requiring substantial programming knowledge (e.g. ARIS and TOVE [4]).

We have been developing a new approach, the Enterprise Modelling Language, based on trees, overlays and fish-eye viewers to overcome the shortcomings of existing workflow notations. EML is the first tree overlay structure visual language in the area of business process modeling. Service architectures are represented as trees and business sequences are modeled as process overlays on the service trees. By combining these two mechanisms EML gives users a clear overview of an enterprise system structure while business processes are modeled by overlays on the same view. EML uses a multi layer structure to model business processes, exception handlers and dependency triggers on different levels. This approach significantly reduces the complexity of business processes. Please refer to [7] for a detailed description of EML.

\section{MaramaEML}

We have developed an integrated design environment, MaramaEML for creating EML specifications. This IDE provides a platform for: efficient EML visual model creation, inspection, editing, and storage, model driven code generation, and integration with other diagram types. Figure 1 shows an overview of whole MaramaEML environment structure. MaramaEML's major features include:

(1) MaramaEML permits efficient production of EML visual models (a) facilitating their creation, display, editing, storage, code generation and integration with other diagrams;

(2) Due to the complexity of business processes, a single modelling notation is insufficient to satisfy all modelling needs. We believe that the ideal solution is to provide the user access to an integrated visual modelling environment. The MaramaEML support tool includes concurrent EML (Figure1 (a)), BPMN ( (b) and Form Chart (c) views, with BPEL scripts (d).

(3) MaramaEML supports automatic BPEL code (d) generation from BPMN, EML and Form-Chart views; this involves model dependency analysis and its translation to structured activity constructs. The form chart view can also be compiled to client-side JavaScript which defines page navigations and clientserver communications.

(4) The multiple modeling notations are integrated together using generated XML-based BPEL scripts as the interchange format. This provides multi-level support for integrating complex enterprise system models.

(5) We have integrated a $3^{\text {rd }}$ party LTSA engine to verify the correctness of generated BPEL code. This engine compiles EML output (BPEL) displaying results in a MaramaEML panel. If there are no compilation errors, an LTS diagram (Labelled Transition System) is shown (e).

(6) A distortion-based fisheye zooming function has been implemented in MaramaEML to enhance complex diagram scalability and navigability. 
MaramaEML is implemented using our Marama meta-tool as a set of Eclipse plug-ins, providing a robust and scalable design tool. We specified the EML, BPMN and Form Chart domain-specific visual language notations and meta-models and generated Eclipse-based editors from these to realise the basic support environment. The tree layout, overlays and distortion-based displays are all implemented as complex visual event handlers. The integration of EML with BPMN notation, code generation of BPEL, and LSTA engine integration are implemented as eventdriven, model-level data updates.

Performance simulation [5] is also incorporated in the integrated MaramaEML, facilitating cost-effective tests of the integrated specifications using random data with visualisation of test results using the same designlevel specification views.

\section{Evaluation}

We have conducted three evaluations for MaramaEML. The first was an extensive cognitive dimensions analysis guiding the design and implementation. It was undertaken by the MaramaEML designer with closeness of mapping and hidden dependency mitigation emphasised. The second was a small scale task-based end-user evaluation of an early release version. The objective was to assess how easy it was to learn to use MaramaEML and how efficiently it can solve the diagram complexity problem as input to refining the software. The third evaluation was a large formal end user evaluation of the most recent release. Feedback suggests MaramaEML is very straightforward to use and understand. Users feel the tree overlay method greatly reduces the complexity of modelling business processes compared to using only conventional BPMN views. The automatic code generation and multi-view collaboration were seen as enhancing the modelling strength. The zooming function is quite easy to use and increases the navigation ability evidently.

\section{References}

[1] BPMI, http://www.ebpml.org/bpml.htm, 2006

[2] C. Marshall, Enterprise Modelling with UML. Designing Successful Software Through Business Analysis, Addison Wesley, 2000

[3] D. Draheim and G. Weber, Form-Oriented Analysis, Springer-Verlag Berlin Heidelberg, 2005

[4] F. Buschmann, R. Meunier, and H. Rohnert, etc, Pattern-Oriented Software Architecture. John Wiley and Sons, 1996

[5] J.C., Grundy, J.G.,Hosking, L., Li and N. Liu, "Performance Engineering of Service Compositions", Proceeding of ICSE2006, p26 p32, Shanghai, China

[6] J. Kornkamol, S. Tetsuya, and T. Takehiro, "A Visual Approach to Development of Web Services Providers/Requestors", Proc. VL/HCC'03 p251 p253

[7] L. Li, J.G. Hosking and J.C. Grundy, EML, A Tree Overlay-based Visual Language for Business Process Modeling, Proceeding of ICEIS 07, 13 19, Funchal, Madeira, Portugal

[8] Martinez, A.; Patino-Martinez, M.; Jimenez-Peris, R.; Perez-Sorrosal, F., ZenFlow: a visual Web service composition tool for BPEL4WS, Proc IEEE VLHCC'05 181-188, 2005

[9] Pautasso, C.; Alonso, G., Visual composition of Web services, Proc IEEE HCC'03. 92-99,. 2003

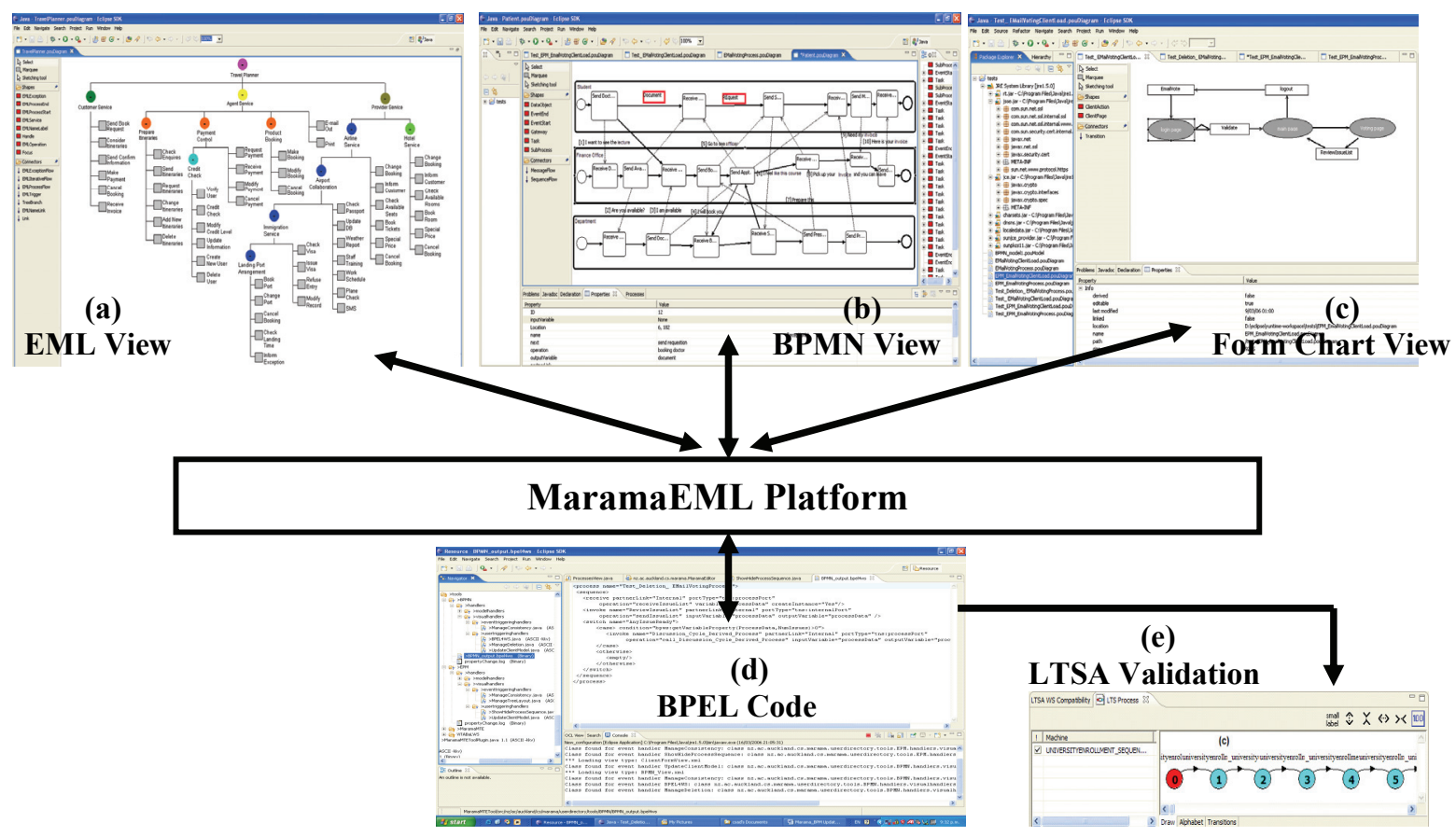

Figure 1. MaramaEML Integrated Environment 
Appendix:

\section{Brief Presentation Description}

\section{Part 1: Brief EML Introduction}

At the start of the presentation, the major concepts and semantic meaning of the Enterprise Modelling Language will be introduced via a set of examples. This will include a short motivation, modelling elements, the tree-overlay structure, process flows, exception handling, service reuse, dependency/internal exceptions, iteration and selection.

\section{Part 2: Detailed MaramaEML Demo}

A detailed software demonstration will be introduced in this part. This will focus on a process modeling scenario with MaramaEML. Key tasks will include:

- $\quad$ extend an existing EML tree; (A1)

- collapse/expand service nodes in an EML tree

- show a process overlay on a service tree (A2)

- $\quad$ show a trigger overlay on a service tree (A3)

- $\quad$ show an exception handling overlay (A4)

- integration of the overlays
- $\quad$ show a BPMN diagram in MaramaEML (A5)

- demonstrate multi-view collaboration (A6)

- demonstrate BPEL code generation (A7)

- demonstrate LTSA validation of BPEL (A7)

- demonstrate fisheye/zooming support (A8)

If time permits, some optional features will be shown

- Form chart modelling

- Form chart diagram compilation to client side javascript

\section{Part 3: Evaluation Results and Discussion}

We will conclude the presentation with evaluation results (A9) and discussion of future work.

\section{Maturity and Availability}

Marama is open source and is available for download - https://wiki.auckland.ac.nz/display/csidst. It has been used to develop a wide range of multi-view, domain-specific visual language tools. MaramaEML is not currently available but will be made available for download in the near future.

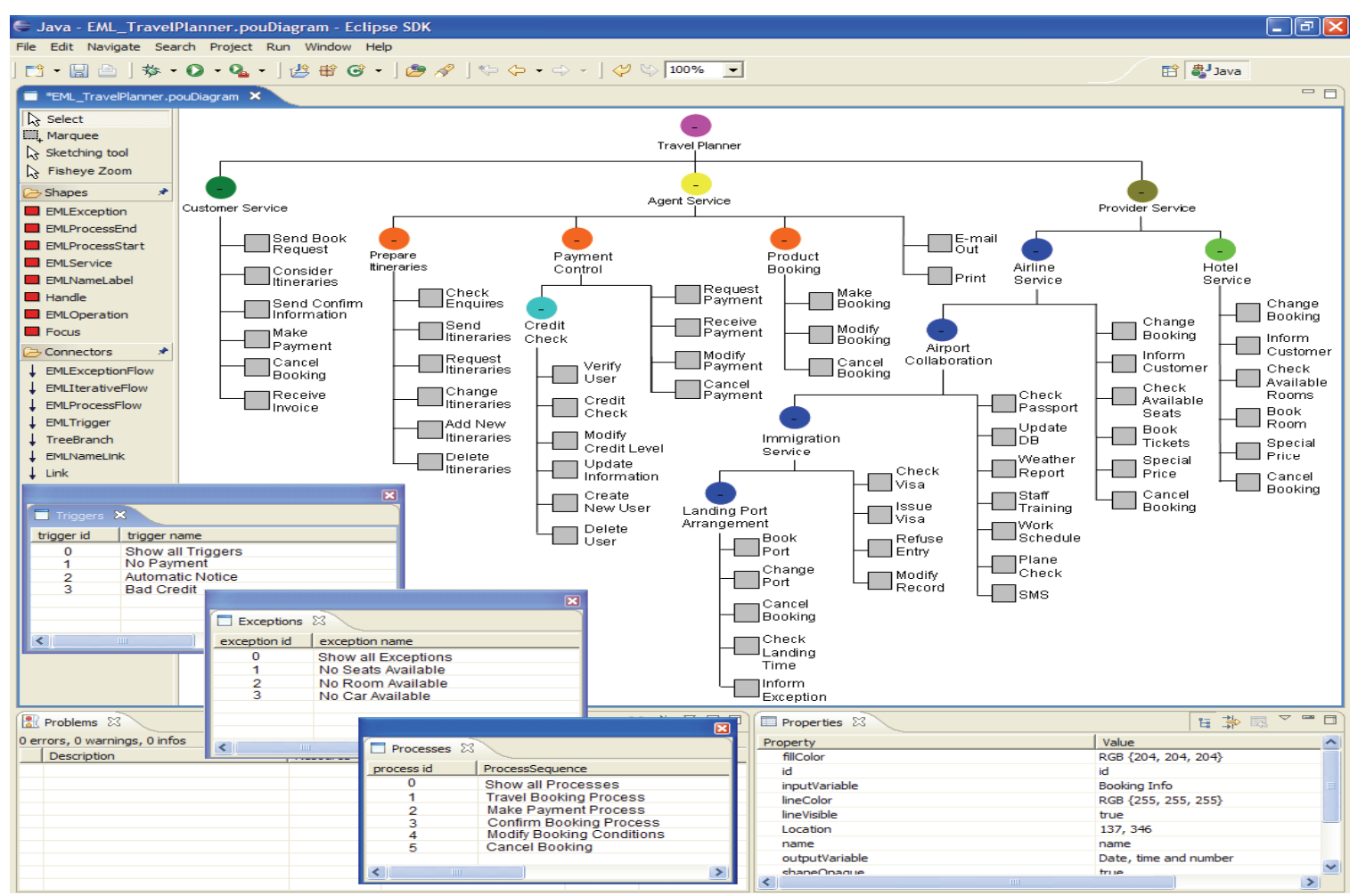

Figure A1: EML Tree in MaramaEML 


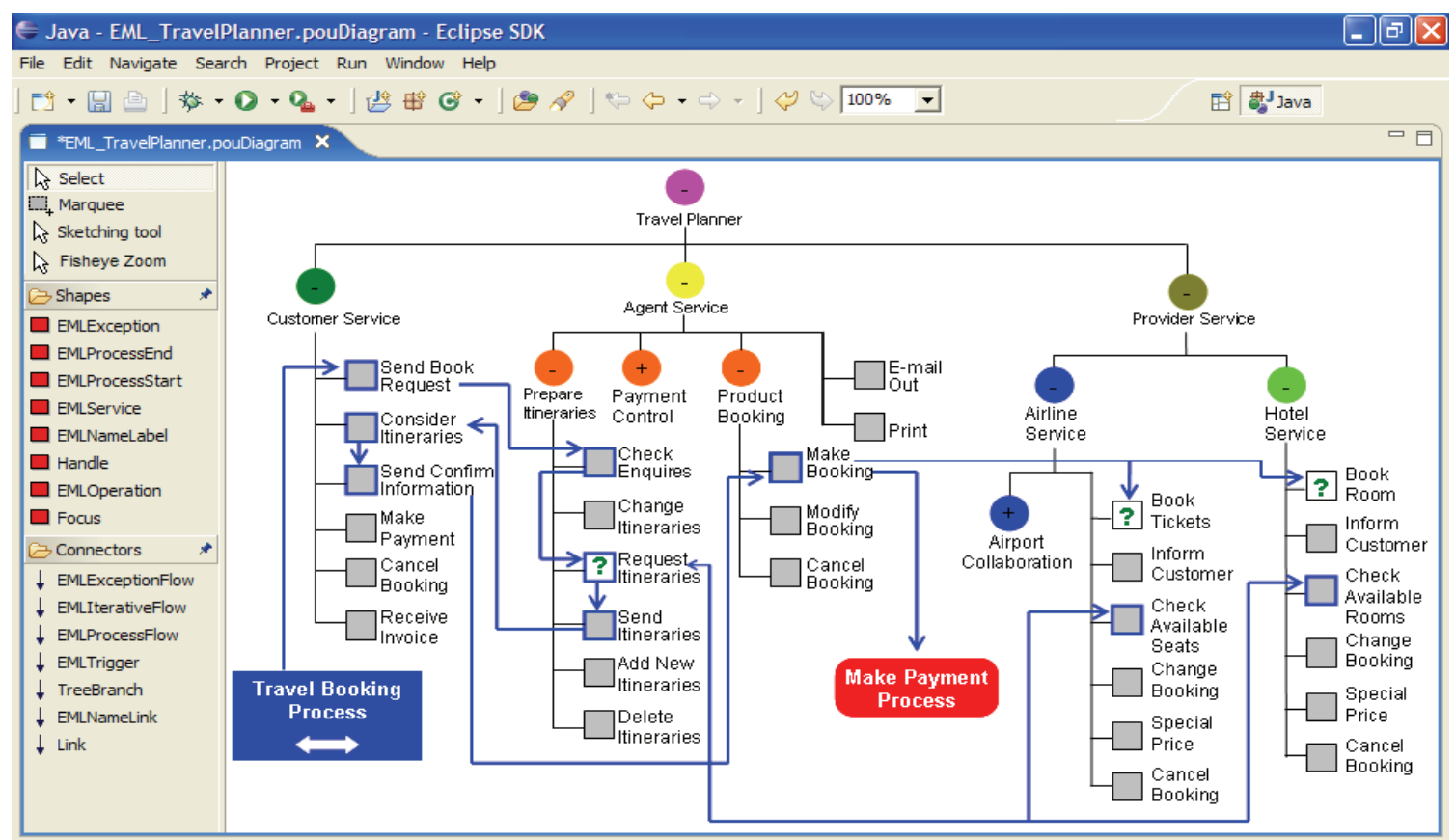

Figure A2: Process Overlay

Java - EML_TravelPlanner.pouDiagram - Eclipse SDK

File Edit Navigate Search Project Run Window Help

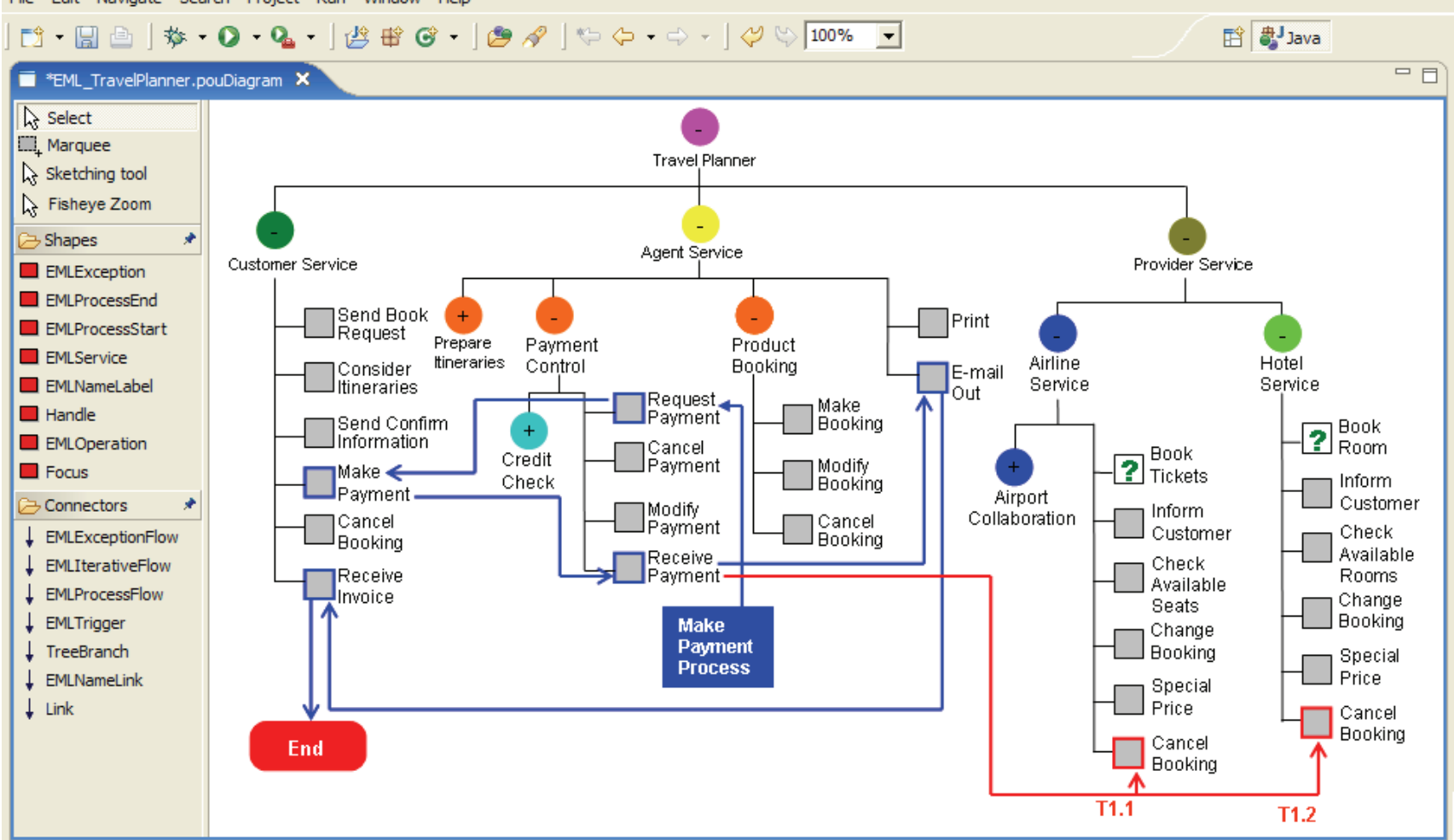

Figure A3: Triggers 
File Edit Navigate Search Project Run Window Help
$[9 \cdot$
落 $-0 \cdot 0$
世
$\Leftrightarrow$

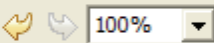
醐Java

E "EML_TravelPlanner.pouDiagram $x$

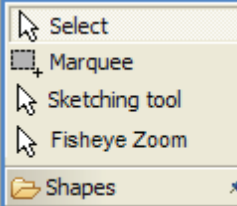

$\square$ EMLException

$\square$ EMLProcessEnd

$\square$ EMLProcessStart

$\square$ EMLService

$\square$ EMLNameLabel

$\square$ Handle

$\square$ EMLOperation

$\square$ Focus

$\rightarrow$ Connectors

$\downarrow$ EMLExceptionFlow

$\downarrow$ EMLIterativeFlow

$\downarrow$ EMLProcessFlow

$\downarrow$ EMLTrigger

$\downarrow$ TreeBranch

$\downarrow$ EMLNameLink

$\downarrow$ Link

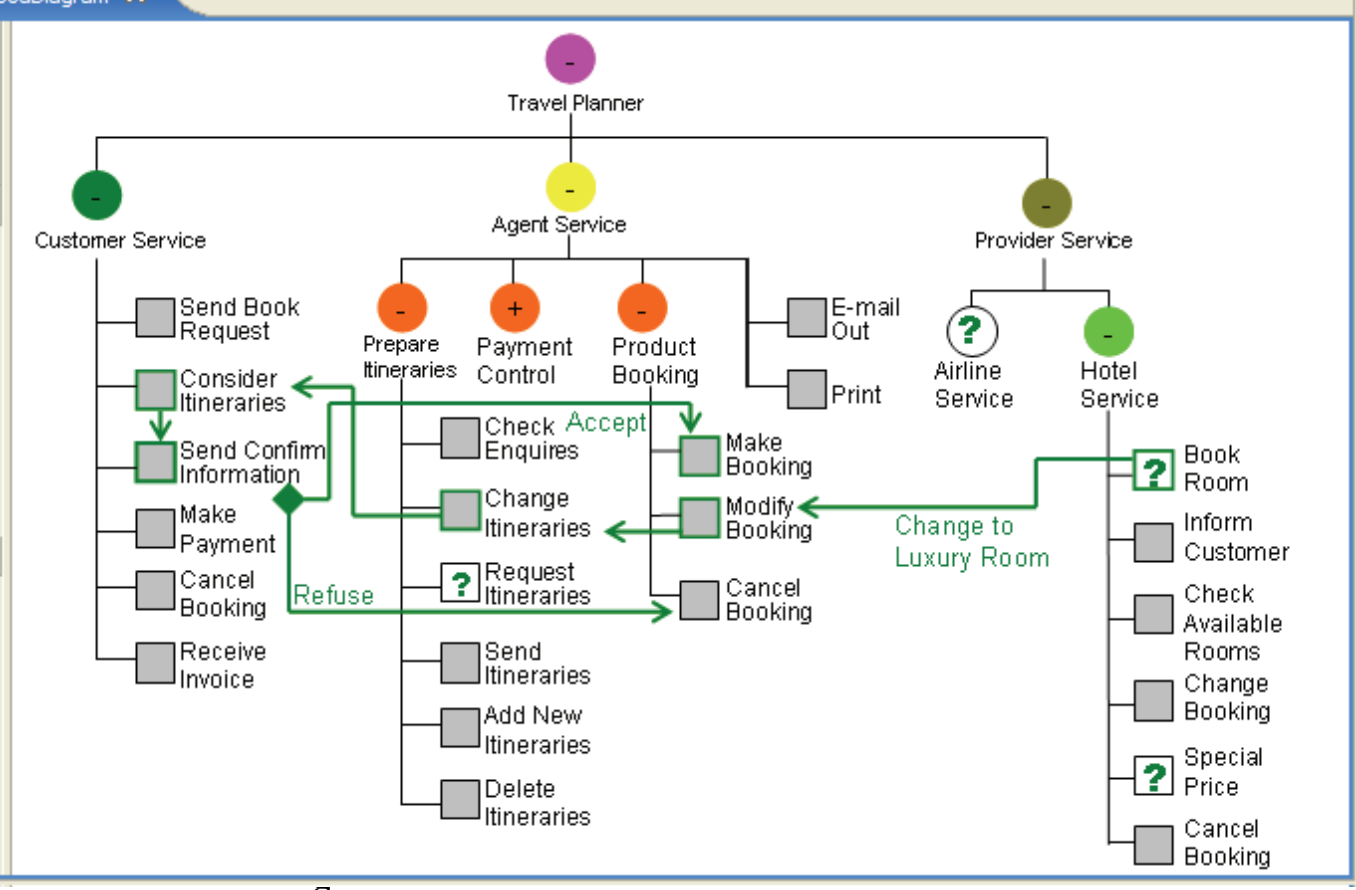

Figure A4: Exception Handling Layer

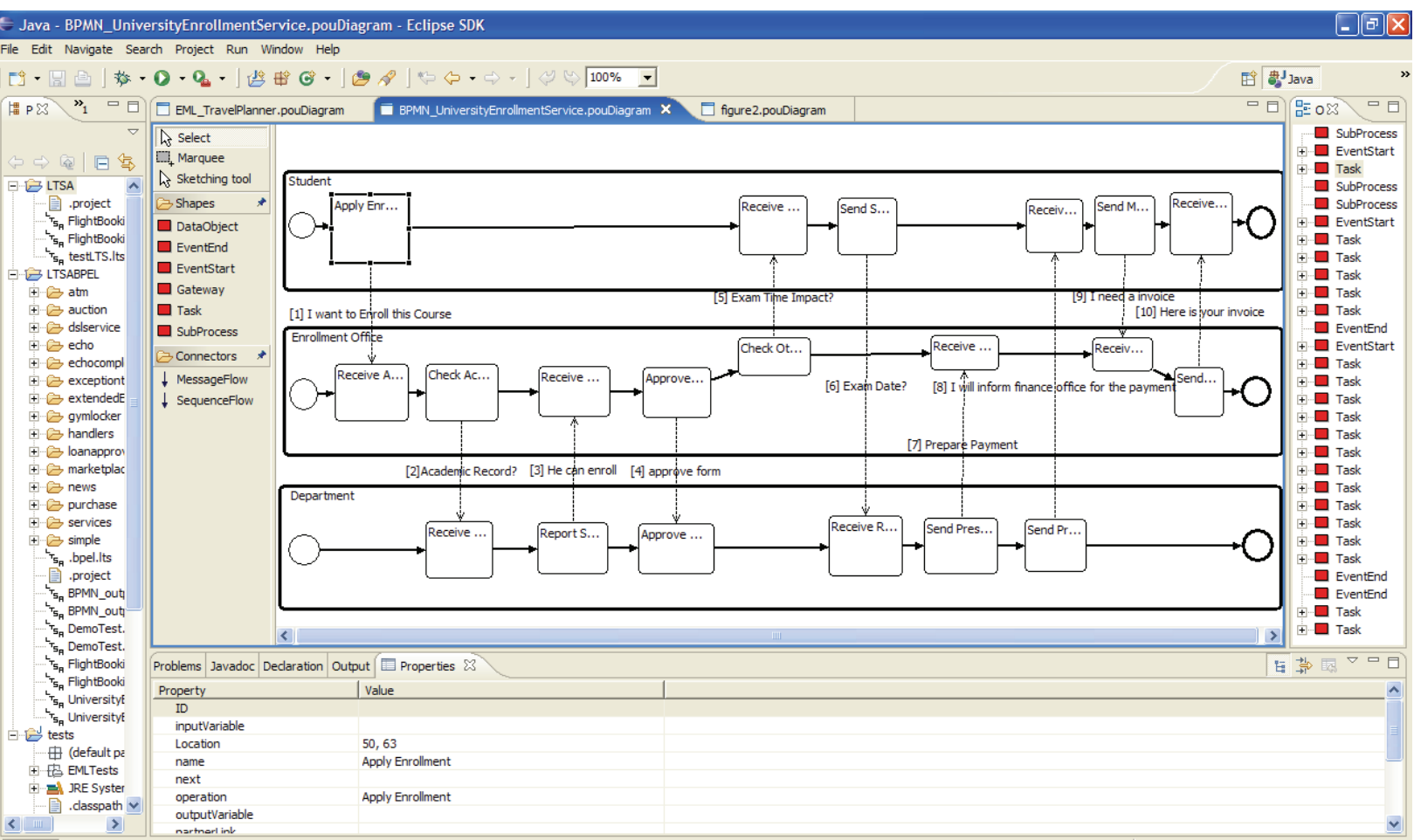

Figure A5: BPMN Diagram in MaramaEML 


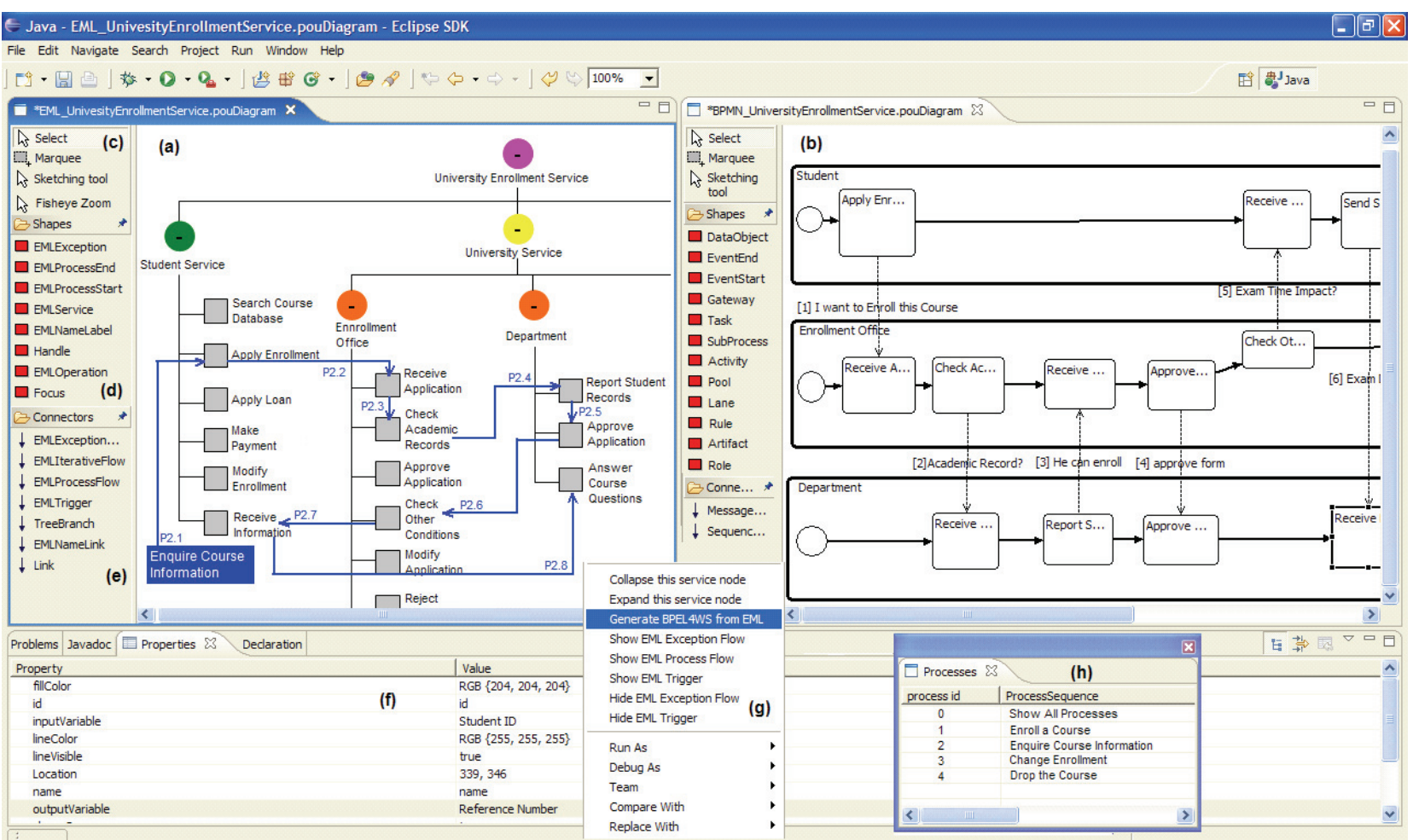

Figure A6: Multi-View Support (EML \& BPMN Collaboration)

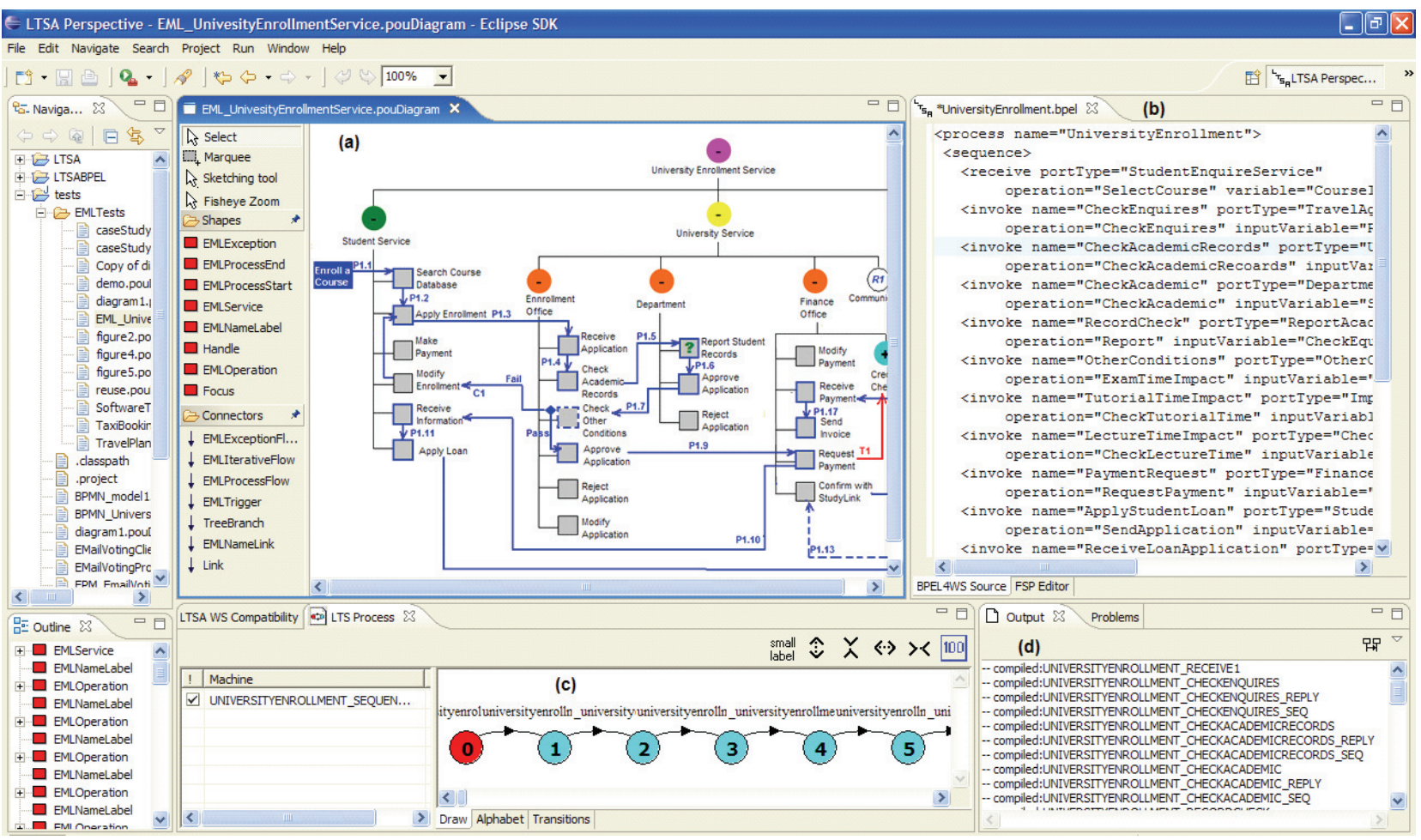

Figure A7: BPEL Code Generation \& LTSA Validation 


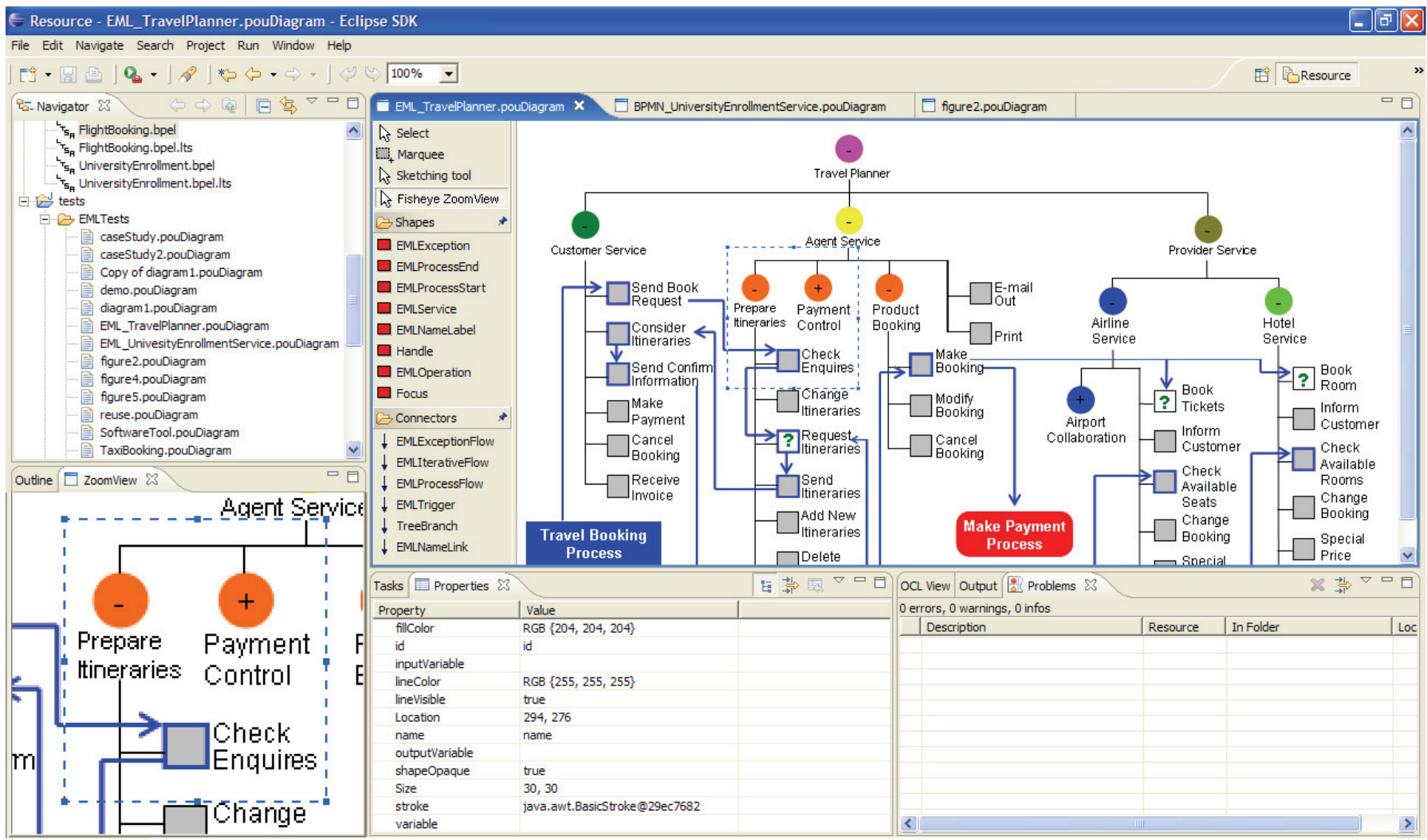

Figure A8: Zoom View Support in EML Tree

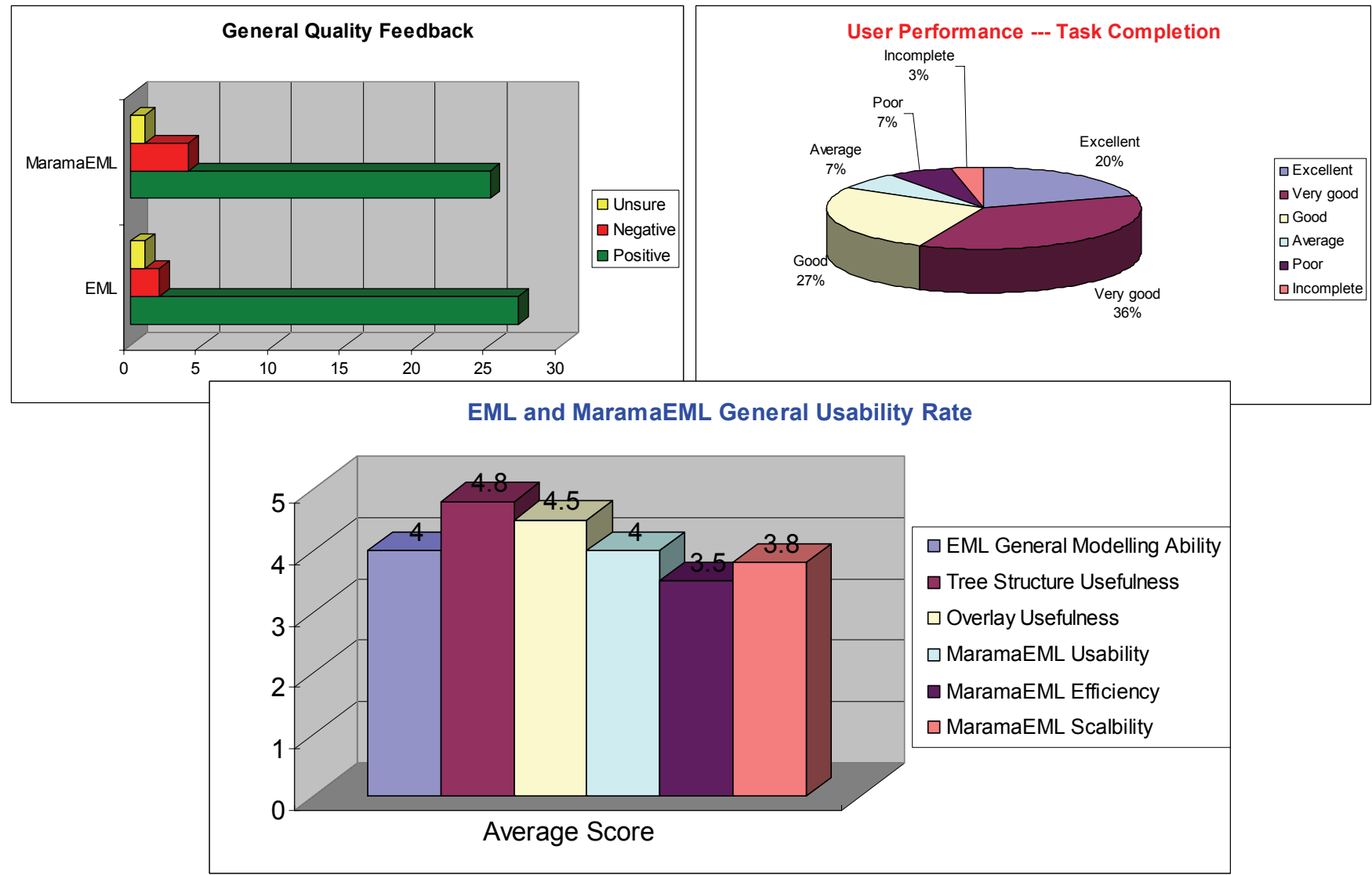

Figure A9: Formal Evaluation Feedback Summary 